\title{
VALOR DE MERCADO EN EL FÚTBOL: ¿ES CÓMO TODOS DICEN?
}

\section{MARKET VALUE INN FOOTBAL: IS LIKE EVERYONE THINKS?}

\author{
Byron Gallegos $^{1}$, Jhon Orrala ${ }^{2}$, Milton Paredes Aguirre ${ }^{3}$
}

\begin{abstract}
Palabras clave: Resumen
El fútbol es el deporte con más aficionados en el planeta y, por ende, Valor de mercado, es de esperarse que se manejen cantidades exorbitantes de dinero. fútbol, regresión lineal desembolsan grandes cantidades de dinero y la pregunta en cuestión múltiple, Conmebol. es: ¿se justifica que el valor de mercado de un jugador sea tan alto?. Por consiguiente, el presente estudio tiene por objetivo identificar cuáles son las variables que afectan al valor de mercado de un futbolista; para este caso en particular, se tomó en consideración una muestra de jugadores del top 35 de equipos de Conmebol en la temporada 2017-2018. Para lograrlo, se aplicó la metodología de regresión lineal múltiple bajo la aplicación de dos modelos. En el primero se tuvo en cuenta sólo variables referentes al desempeño. En el segundo modelo se incluyeron variables de naturaleza externa. Los resultados revelaron las variables que explican mayormente el valor de mercado de un jugador. A su vez, se aplicaron varias pruebas de validación al modelo final, las cuales demostraron su efectividad. Finalmente, se concluye que el grado de influencia que poseen los jugadores también constituye un factor determinante en el valor de mercado.
\end{abstract}

Códigos JEL: J31, J44

\footnotetext{
${ }^{1}$ Escuela Superior Politécnica del Litoral, ESPOL, Facultad de Ciencias Sociales y Humanísticas, Campus Gustavo Galindo Km. 30.5 Vía Perimetral, P.O. Box 09-01-5863, Guayaquil, Ecuador. E-mail: bxgalleg@espol.com.ec

${ }^{2}$ Escuela Superior Politécnica del Litoral, ESPOL, Facultad de Ciencias Sociales y Humanísticas, Campus Gustavo Galindo Km. 30.5 Vía Perimetral, P.O. Box 09-01-5863, Guayaquil, Ecuador. E-mail: jodaorra@espol.com.ec

${ }^{3}$ Escuela Superior Politécnica del Litoral, ESPOL, Facultad de Ciencias Sociales y Humanísticas, Campus Gustavo Galindo Km. 30.5 Vía Perimetral, P.O. Box 09-01-5863, Guayaquil, Ecuador. E-mail: isparede@espol.edu.ec
} 


\begin{abstract}
Keywords:
Abstract

Market value,

Football is the sport with more fans on the planet and therefore, it is

soccer,

Multiple linear

regression,

Conmebol.

expected that exorbitant amounts of money will be handled.

Particularly when signing players, football clubs disburse large amounts of money and the question in question is: it is justified that the market value of a player is so high?. Therefore, the objective of this study is to identify the variables that affect the market value of a soccer player; For this case, a sample of players from the top 35 of Conmebol teams in the 2017/2018 season was taken into consideration. To achieve this, the multiple linear regression methodology was applied under the application of two models. In the first, only variables related to performance were considered. In the second model, variables of an external nature were included in the first model. The results revealed the variables that mostly explain the market value of a player. In turn, various validation tests were applied to the final model, which demonstrated its effectiveness. Finally, it is concluded that the degree of influence that the players possess also constitutes a determining factor in the market value.
\end{abstract}

JEL Codes: J31, J44

\section{INTRODUCCIÓN}

En años recientes el mercado de transferencias de jugadores de fútbol masculino a nivel mundial se ha desarrollado a tal punto que, cada vez se invierten mayores montos para la inclusión de nuevos talentos y, a su vez, se ha vuelto común el hecho de que en cada periodo de transferencias se establezcan récords (Clark, 2010). Esta situación ha situado al mercado en una posición inestable e inflacionaria; y todo esto ha llevado a que distintos investigadores se pregunten si estas cifras están justificadas en un análisis objetivo de factores o si simplemente, se basan en la especulación de precios (He, et al., 2015).

Con respecto a lo anteriormente planteado, el problema radica en la escasez de herramientas objetivas para la valoración de jugadores a nivel de Sudamérica. La implementación de modelos objetivos basado en el análisis de datos permite a los clubes de fútbol conocer la valoración de los jugadores de tal manera que eviten caer esencialmente en la sobreestimación (Swanepoel, 2016). Cabe mencionar que, existen estudios afines con enfoques econométricos, sin embargo, el objetivo de estos es contribuir en el proceso de decisión de inversión a nivel gerencial (Majewski, 2016).

\section{REVISIÓN DE LITERATURA}

Dada la relevancia del tema, existen algunos estudios al respecto. Uno de estos analiza la valoración de mercado de diferentes jugadores de La Liga (España) a lo largo de la temporada 2014/2015, basándose en datos provenientes de páginas reconocidas como: Transfer Market, WhoScored, entre otros. En tal estudio se emplean regresiones LASSO con el fin de estimar una ecuación para el valor de mercado y una para el rendimiento. Luego de esto, compara las estimaciones con los valores reales de jugadores transferidos en esa temporada para finalmente 
verificar si estos se encontraban sobrevalorados o infravalorados; dicho fenómeno se atribuyó a una variable no estudiada que representaba la atención mediática (He, et al., 2015).

De la misma manera, el CIES (Centre International d'Etude du Sport) Football Observatory, presentó un estudio en el año 2018 con un enfoque científico a fin de evaluar bajo una perspectiva predictiva el valor de transferencia de los jugadores profesionales. Este estudio afirma que el poder explicativo del enfoque econométrico sugiere un alto grado de racionalidad del mercado de transferencias de fútbol y que, además, la mayor parte de las transferencias sigue una lógica previsible que puede ser modelada con fines de pronóstico. Los autores establecen que el principal desafío de su modelo es poder anticipar el nivel de inflación debido a la dinámica del mercado de pases, porque la inflación no interviene de manera lineal en el tiempo o según los segmentos del mercado. No obstante, concluyen que esto no es relevante para la especificación del modelo porque las determinantes del precio son estables (Poli, et al., 2018).

Una investigación relevante con respecto a la valoración de jugadores de fútbol fue realizada en el año 2013. El estudio, consiste en estimar mediante un modelo econométrico el valor de mercado de los jugadores de fútbol de la Bundesliga basado en variables que se dividen en dos grupos: variables que hacen referencia al talento y variables externas o exógenas que también afectan al valor de mercado del jugador. Una vez estimado el valor de mercado, los autores comparan el valor estimado del modelo con el valor propuesto por la página "transfermarkt.de". Finalmente, concluyen que las variables que tomaron en cuenta para la investigación fueron significativas para explicar y estimar el valor de mercado y que, el valor de mercado de los jugadores sugerido en el portal web de "transfermarkt.de" y el estimado por el modelo econométrico, son parecidos (Herm, et al., 2014).

En esta misma línea, otra investigación importante y actual (año 2019), fue realizada por Julian Hofmann y otros, cuya finalidad es analizar el valor de mercado de jugadores de fútbol profesionales y determinar cuáles factores afectan mayormente a la valoración de dicho jugador en el mercado, ya que ellos se planteaban antes del estudio, conocer la importancia de la popularidad para un jugador y su valor en el mercado. Dentro de sus conclusiones, se encuentran que efectivamente la popularidad sí afecta significativamente a la valoración del jugador y que, de hecho, es más significativa que variables referentes al talento (Hofmann, et al., 2019).

Otro trabajo importante referente a la valoración objetiva de jugadores mediante análisis estadístico es el realizado por Oliver Müller, Alexander Simons y Markus Weinmann en 2017, quienes en su trabajo contribuyen al tema identificando la deficiencia que existe en las valoraciones basadas en la multitud (como, por ejemplo, las valoraciones que dan comunidades en línea a los jugadores). También sintetizan la investigación académica que refleja las variables que deben ser tomadas en cuenta a la hora de valorar jugadores en el mercado de pases de fútbol, usan una muestra grande de jugadores de las 5 grandes ligas europeas y evalúan la precisión de su modelo comparándolo con los valores reales de algunas transferencias. Finalmente, comparan los resultados con las tasas reales de transferencias y concluyen que el modelo es más exacto en el $90 \%$ de los casos, donde las tasas de transferencias son bajas y que el $10 \%$ que hace referencia a tasas altas, es mejor estimado por el juicio de las comunidades online (Müller, et al., 2017).

De todo lo mencionado, en este estudio se tomará en consideración principalmente las investigaciones realizadas por Herm (2014) y Müller (2017), ya que marcan una ruta importante a seguir en esta línea de investigación debido a la objetividad de sus metodologías.

\section{MATERIALES Y MÉTODOS/ METODOLOGÍA}

Para la recolección de datos, se recopiló información de los jugadores de los 35 mejores equipos a nivel de Conmebol según el ranking FIFA (índice que considera desempeño histórico local e internacional), mediante una extracción directa proveniente de diferentes paginas 
especializadas, desde los principales portales de valoración futbolística (Tranfermarkt y WhoScored) con el objetivo de extraer las variables de desempeño, hasta información proveniente de redes sociales con la finalidad de valorar el impacto mediático.

La razón para no haber escogido la muestra de manera aleatoria es debido a la limitación de acceso a la información, ya que al tener cada equipo la misma probabilidad de ser escogidos para el estudio, muchos de los equipos que se escogían eran equipos de poca importancia en el contexto local e internacional, de los cuales, el acceso a la información de sus jugadores era bastante limitada. De los 10 países que conforman Conmebol, el único que no formó de la muestra es Venezuela, debido a que no apareció dentro del top 35.

El número de observaciones tomadas para este estudio fue de 144 jugadores, de los cuales 48 son ofensivos, 48 son mediocentros y 48 son defensores. Estas observaciones pertenecen a jugadores que hayan jugado al menos un partido en la temporada 2017/2018. Es necesario considerar que el análisis de desempeño de los jugadores varía dependiendo de su posición, ya que mientras a los atacantes se les exige que hagan goles y asistan, a los mediocentros se les pide que tengan una mayor cantidad de pases precisos y a los defensas se los evalúa según el número de duelos ganados.

El modelo que se plantea (apéndice 1) probar consta de 20 variables explicativas y como se ha venido mencionando a lo largo de este capítulo, se trata de un modelo de regresión lineal múltiple que se puede dividir en dos especificaciones a fin de probar el impacto por un lado de las variables de desempeño y, por otro lado, el modelo que contempla la inclusión de variables externas.

A este modelo al que se le aplicará todas las pruebas con el objetivo de encontrar un modelo que cumpla con todas las condiciones que se mencionarán en las siguientes secciones. (apéndices 2, 3 y 4).

\section{RESULTADOS}

Luego de estimar los respectivos modelos, como se detalla en el apéndice 5 donde se reflejan los resultados obtenidos de ambas estimaciones bajo el modelo de regresión planteado, se concluyó por un lado, del modelo 1 , se puede decir que el $\mathrm{R}$ cuadrado tiene un valor de 0.205; es decir, las variables que hacen referencia al desempeño del jugador para este caso en particular, explican sólo el $21 \%$ de la varianza del valor de mercado, mientras que cuando se incluyeron las variables externas al modelo, el $\mathrm{R}$ cuadrado sube a casi $70 \%$, por lo cual se pudo deducir que como lo sugiere la teoría, las variables que son ajenas al desempeño del jugador terminan explicando mayormente su valor de mercado.

Otro punto importante, es que, en el primer modelo, las variables de desempeño que resultaron estadísticamente significativas fueron: goles, precisión, flexibilidad y equipos pasados y de estas 4 variables, al momento que se incluyeron las variables externas en el modelo, 2 dejan de ser significativas (precisión y flexibilidad) y; en el modelo general, el valor de mercado se vio explicado por dos variables de desempeño y dos externas.

Estos resultados sugirieron que el mejor modelo debería de contener las cuatro variables significativas del modelo 2 , como se detalla la estimación en el apéndice 6.

\section{CONCLUSIONES}

La importancia del presente trabajo radica en el marco económico, ya que el mercado de traspasos futbolísticos es un mercado en el que se mueven cantidades exorbitantes de dinero cada año, tanto a nivel de Sudamérica y del mundo; y, por lo tanto, es importante analizar qué variables son las que explican estos valores de mercado que en algunos casos son excesivamente altos e injustificados (Lago \& Martín, 2007).

Luego de analizar los resultados obtenidos en el presente estudio, se concluye que los resultados van acordes a lo que sugiere la literatura, aunque con ciertas diferencias; lo cual se esperaba ya que 
se trata de un estudio de carácter exploratorio y, de hecho, el presente trabajo es uno de los primeros que se ha realizado a nivel de Sudamérica con un enfoque cuantitativo.

Las principales conclusiones de manera general son: que se logró identificar cuáles son las variables que afectan mayormente al valor de mercado mediante un enfoque objetivo, se logró identificar cuál es el modelo que mejor explica el comportamiento del valor de mercado, se logró comparar los resultados estimados del modelo con los valores propuestos por la plataforma transfermarkt y finalmente, se pone a disposición estos hallazgos a futuros investigadores que estén interesados en desarrollar mucho más el tema ya que este estudio puede servir como punto de partido debido a su naturaleza de carácter exploratorio donde se obtuvieron resultados bastante interesantes.

El valor agregado del presente trabajo se puede simplificar en dos aristas: la primera es que es el primer estudio que se realiza a nivel sudamericano con un enfoque cuantitativo y la segunda es que es el primer estudio que propone una variable como número de seguidores en redes sociales para analizar su significancia estadística en un modelo que busque predecir el valor de mercado.

Las principales fortalezas de este estudio son que: el modelo final tal como se muestra en los resultados es robusto, cumple con los supuestos de Mínimos Cuadrados Ordinarios y el nivel predictivo del mismo es bastante aceptable, teniendo en cuenta que, como todo modelo, este no es más que una simplificación de la realidad.

Las principales limitaciones de este son que: al ser tan complicado recolectar datos, no fue posible realizar un muestreo aleatorio y esto limita seriamente la interpretación de este, ya que como se mencionó en la sección anterior, los resultados de este estudio no son extrapolables y corresponden sólo a la muestra que se analizó que son equipos del top 35 del ranking de Conmebol y esto limita en gran medida el alcance del estudio. Es decir, estos resultados hacen se pueden llegar a cumplir en equipos con características similares.

Otra limitación importante por considerar es que se trata de un modelo predictivo no causal, por lo que se debe tener especial cuidado con la interpretación de los resultados.

Finalmente, para futuras investigaciones, se recomienda buscar (si es que existe) una base de datos centralizada donde se puedan obtener diversos datos de los jugadores, expandir el alcance de este tipo de estudios hacia los arqueros (quienes se analizan bajo una perspectiva distinta) y analizar el contexto nacional a fin de conocer cómo se comporta el mercado ecuatoriano con respecto al del resto del continente.

\section{AGRADECIMIENTOS}

Agradecemos particularmente a nuestro tutor de tesis, el Ing. Milton Paredes por todo el apoyo brindado a lo largo de la materia integradora, al Econ. Ronald Campoverde por sus comentarios que fueron fundamentales en el desarrollo de nuestro proyecto y al Econ. Gonzalo Sánchez por sus comentarios brindados al inicio del proyecto, los cuales nos sirvieron como guía para saber qué camino tomar con respecto al tema que hemos desarrollado.

\section{REFERENCIAS}

Clark, J., 2010. Back on Track? The Outlook for the Global Sports Market to 2013, London: s.n.

He, M., Cachucho, R. \& Knobbe, A., 2015. Football Player's Performance and Market Value. Conference: Proceedings of the 2nd workshop of sports analytics, European Conference on Machine Learning and Principles and Practice of Knowledge Discovery in Databases.

Herm, S., Callsen-Bracker, H.-M. \& Kreis, H., 2014. When the crowd evaluates soccer players' market values: Accuracy and evaluation attributes of an online community. Sport Management Review, noviembre, 17(4), pp. 484-492. 
Hofmann, J., Schnittka, O., Johnen, M. \& Kottemann, P., 2019. Talent or popularity: What drives market value and brand image for human brands? Journal of Business Research, mayo.

Lago, C. \& Martín, R., 2007. Determinants of possession of the ball in soccer. Journal of Sports Sciences, 25(9).

Majewski, S., 2016. Identification of Factors Determining Market Value of the Most Valuable Football Players. Journal of Management and Business Administration, 24(3).
Müller, O., Simons, A. \& MarkusWeinmann, 2017. Beyond crowd judgments: Data-driven estimation of market value in association football. European Journal of Operational Research, 1 diciembre, 236(2), pp. 611 $-624$.

Poli, R., Ravenel, L. \& Besson, R., 2018. Scientific assessment of football players' transfer value, s.l.: s.n.

Swanepoel, M., 2016. The Correlation Between Player Valuation and The Bargaining Position Of Clubs In The English Premier League (Epl). International Journal of Economics And Finance Studies, 8(1). 


\section{APÉNDICES}

\section{Apéndice 1: El Modelo Planteado}

$$
Y_{i}=\beta_{0}+\beta_{1} X_{1}+\beta_{2} X_{2}+\beta_{3} X_{3}+\beta_{4} X_{4}+\cdots+\beta_{15} X_{15}+\beta_{16} X_{16}+\cdots+\beta_{20} X_{20}+\varepsilon_{i}
$$

Tal que:

\begin{tabular}{|l|l|l|}
\hline Variables & Descripción & Desempeño/externa \\
\hline$X_{1}:$ Edad & Años de vida & Desempeño \\
$X_{2}:$ Altura & Medido en centímetros & Desempeño \\
$X_{3}:$ Peso & Medido en kilos & Desempeño \\
$X_{4}:$ Precisión & Pases acertados/Media en su posición & Desempeño \\
$X_{5}:$ Goles & Goles/Media en su posición & Desempeño \\
$X_{6}:$ Asistencias & Asistencias/Medias en su posición & Desempeño \\
$X_{7}:$ Flexibilidad & Habilidad para jugar con ambos pies & Desempeño \\
$X_{8}:$ Tiempo en cancha & Número de minutos jugados en la temporada & Desempeño \\
$X_{9}:$ Faltas & Número de tarjetas/Media en su posición & Desempeño \\
$X_{10}:$ Trofeos & Número de títulos ganados & Desempeño \\
$X_{11}:$ Lesiones & Número de partidos perdidos por lesión & Desempeño \\
$X_{12}:$ Jugado en Europa & Si el jugador ha jugado o no en europa & Desempeño \\
$X_{13}:$ Seleccionado & Si el jugador ha sido seleccionado o no & Desempeño \\
$X_{14}:$ Posición & Zona del campo en la que se desempeña & Desempeño \\
$X_{15}:$ Equipos pasados & Número de equipos en los que ha jugado & Desempeño \\
$X_{16}:$ Nacionalidad & País de origen & Externa \\
$X_{17}:$ Nivel económico del \\
club & Valoración económica del club & Externa \\
$X_{18}:$ Atención mediática 1 & Enlaces en google relacionados al jugador & Externa \\
$X_{19}:$ Seguidores & Número de seguidores en redes sociales & Externa \\
$X_{20}:$ Duración del contrato & Vanos que faltan para que finalice el contrato & Externa \\
$Y_{i}:$ Valoración & & Dependiente \\
\hline
\end{tabular}


Apéndice 2: Prueba de Linealidad

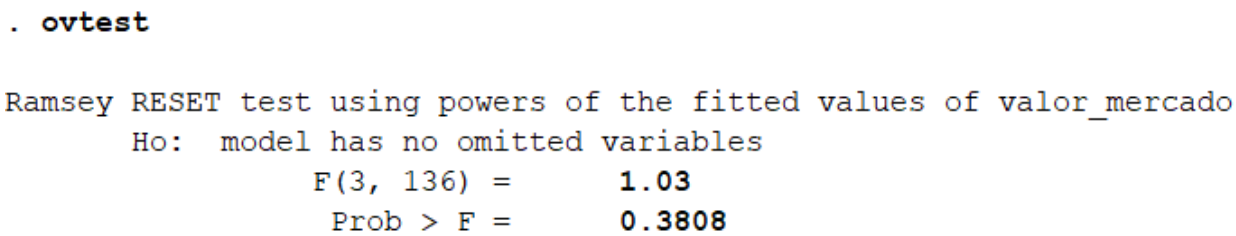

Apéndice 3: Prueba de Normalidad

Normalidad de Residuos

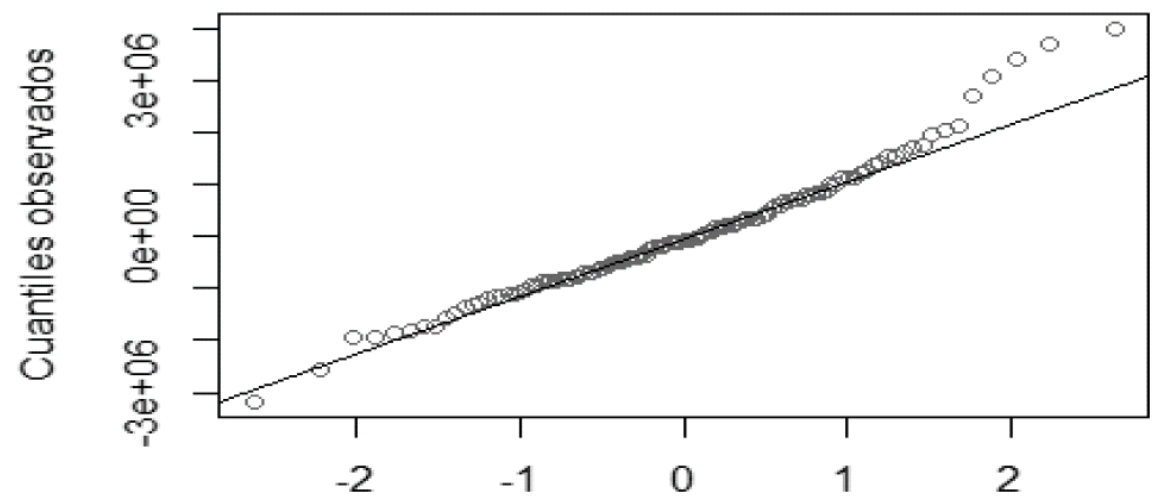

Apéndice 4: Validación Cruzada

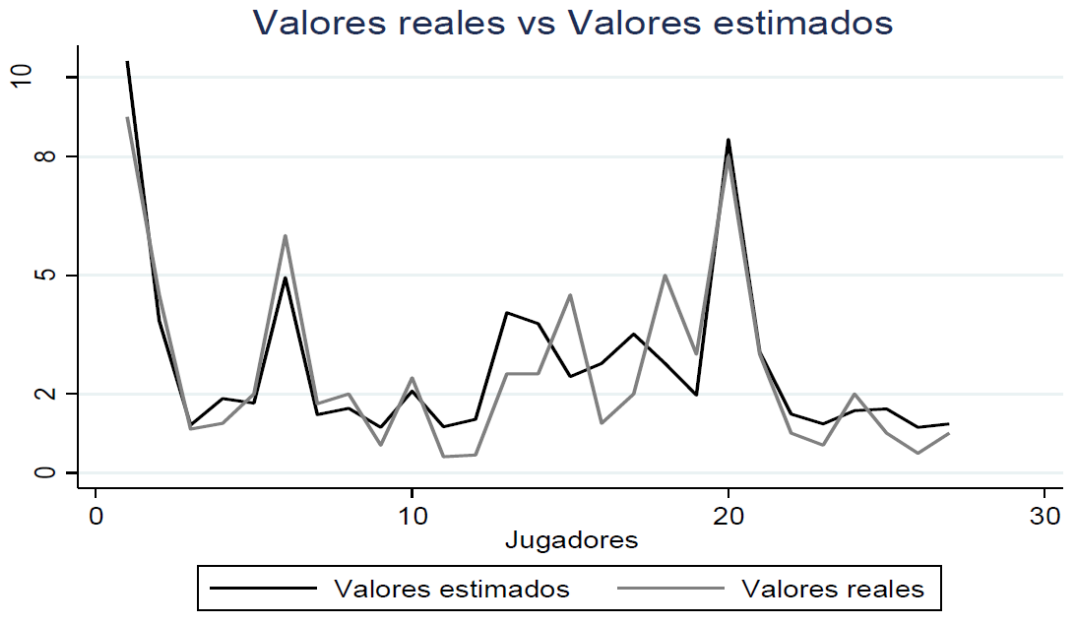




\section{Apéndice 5: Modelos de Regresión}

\begin{tabular}{|c|c|c|}
\hline & Modelo 1 & Modelo 2 \\
\hline VARIABLES & $\begin{array}{c}\text { MCO, Robusto S.E. } \\
\text { (Desempeño) }\end{array}$ & $\begin{array}{c}\text { MCO, Robusto S.E. } \\
\text { (General) }\end{array}$ \\
\hline Edad & 12,487 & $-19,418$ \\
\hline Altura & $-830,510$ & 1821788 \\
\hline Peso & $-1,043$ & $-27,253$ \\
\hline Precisión & $658,3430^{* * *}$ & 1404616 \\
\hline Goles & $427,630 * * *$ & $171,103 *$ \\
\hline Asistencias & 249,091 & 59,037 \\
\hline Flexibilidad & $-128,6941^{*}$ & $-379,323$ \\
\hline Tiempo en Cancha & -425.0 & 44.76 \\
\hline Faltas & 57,099 & $-176,707$ \\
\hline Trofeos & 146,919 & 85,403 \\
\hline Lesiones & 9,236 & -804.7 \\
\hline Jugado en Europa & 337,625 & 130,374 \\
\hline Seleccionado Nacional & $-533,715$ & 341,964 \\
\hline Posición & 182,273 & 65,803 \\
\hline Equipos Pasados & $-159,319 *$ & $-100,533^{*}$ \\
\hline Nacionalidad & & $-57,845$ \\
\hline Nivel Económico & & $0.0220 * * *$ \\
\hline Atención Mediática & & 0.402 \\
\hline Número de Seguidores & & $2.634 * * *$ \\
\hline Duración del Contrato & & 150,619 \\
\hline Constante & $-251,6979$ & $-1408,524$ \\
\hline Observaciones & 144 & 144 \\
\hline R-cuadrado & 0.205 & 0.689 \\
\hline
\end{tabular}

$* * * \mathrm{p}<0.01, * * \mathrm{p}<0.05, * \mathrm{p}<0.1$

Elaboración: Por los autores 
Apéndice 6: Modelos de Regresión

VARIABLES

Modelo 3

\begin{tabular}{ll}
\hline Goles & $153,191^{*}$ \\
& $(81,464)$ \\
Equipos Pasados & $-120,557^{* * *}$ \\
& $(40,410)$ \\
Nivel Económico & $0.0234 * * *$ \\
& $(0.00373)$ \\
Número de Seguidores & $3.074 * * *$ \\
& $(0.439)$ \\
Constante & $1,334,780^{* * *}$ \\
& $(229,121)$ \\
\hline Observaciones & $\mathbf{1 4 4}$ \\
R-cuadrado & 0.658 \\
\hline
\end{tabular}

$* * * \mathrm{p}<0.01, * * \mathrm{p}<0.05, * \mathrm{p}<0.1$

Elaboración: Por los autores 